\title{
Anatomical variations and morphometric properties of the circulus arteriosus cerebri in a cadaveric Malawian population
}

\author{
C. Nyasa1'D, A. Mwakikunga1, 2, L.H. Tembo1,2, C. Dzamalala1', A.O. Ihunwo²® \\ ${ }^{1}$ Biomedical Sciences Department, Anatomy Division, College of Medicine, University of Malawi, Blantyre, Malawi \\ ${ }^{2}$ School of Anatomical Sciences, Faculty of Health Sciences, University of the Witwatersrand, Parktown, Johannesburg, \\ South Africa
}

[Received: 27 October 2020; Accepted: 17 November 2020; Early publication date: 5 December 2020]

Background: Knowledge of the anatomy of the circulus arteriosus cerebri (CAC) is important in understanding its role as an arterial anastomotic structure involved in collateral perfusion and equalisation of pressure, and may explain observed variations in neurovascular disease prevalences across populations. This study was aimed at understanding the anatomical configuration and morphometric properties of the CAC in Malawian population.

Materials and methods: Brains were collected from 24 recently-deceased black Malawian human cadavers during medico-legal autopsies. Photographs of the CACs were taken using a camera placed at a $30 \mathrm{~cm}$ height from the base of the brain. Whole-circle properties and segmental vessel parameters were analysed using the OSIRIS computer programme, paying attention to completeness, typicality, symmetry, and segmental vessel diameters and lengths.

Results: The complete-circle configuration was found in $69.57 \%$ of the CACs. Of these, $37.5 \%$ were typical, representing an overall typicality prevalence of $26.09 \%$. Vessel asymmetry was observed in 30.43\% of cases. There were 7 cases of vessel aplasia and 12 cases of vessel hypoplasia. The posterior communicating artery (PCOA) was the most variable (with 12 variations), widest $(7.67 \mathrm{~mm})$ and longest $(27.7 \mathrm{~mm}$ ) vessel while the anterior communicating artery (AcoA) was the shortest $(0.78 \mathrm{~mm})$. Both the AcOA and the PCOA were the narrowest vessels $(0.67 \mathrm{~mm})$ in this study. CAC variations in Malawian populations appeared to be similar to those observed in diverse populations.

Conclusions: Anatomical variations of the CAC exist in Malawian population and should be taken into consideration in clinical practice. (Folia Morphol 2021; 80, 4: 820-826)

Key words: anatomical configuration, neurovascular diseases, posterior communicating artery, anterior communicating artery, circle of Willis, hypoplasia, aplasia, vessel asymmetry, internal carotid artery 


\section{INTRODUCTION}

The circulus arteriosus cerebri (CAC), also known as the circle of Willis, is a polygonal anastomotic system of arteries that constantly supplies blood to the brain. First described in the year 1658 and later detailed by Thomas Willis in the year 1664 [1], the CAC connects the four sources of brain vascular supply: 2 internal carotid arteries (ICA) and 2 vertebral arteries [19]. In a typical CAC, the ICA give rise to the anterior cerebral arteries (ACA) which are connected to each other by the anterior communicating artery (AcoA) while the vertebral arteries terminate by fusion into the basilar artery [21]. The basilar artery bifurcates to give rise to two posterior cerebral arteries (PCA). The PCA are connected to the ICA system by the posterior communicating arteries (PCOA) [1]. This anastomotic network forms an arterial ring at the base of the brain, which serves to provide collateral blood circulation to the brain through the major branches that arise from it and equalize the pressure. Anastomotic flow of blood in the proximal portions of the cerebral arterial tree is of great physiological importance, in as far as adequate brain perfusion is concerned. This is most evident in times of arterial incompetence such as during states of occlusive cerebrovascular conditions [21].

The clinical significance of the CAC needs no special emphasis. A number of studies have established the role of an anatomically complete circle of Willis in maintaining sufficient cerebral perfusion $[19,20]$. Variations in the anatomy of the CAC have also been observed across population groups, ages and genders, some of which highly correlate with increased risk of neurosurgery complication and conditions such as migraine $[1,10]$, stroke [22, 24], and aneurysms $[15,23]$. Evidence reveals existence of both typical and atypical CAC variants within populations [4]. A typical CAC exhibits a complete polygonal structure with all its component vessels intact and branching from usual locations. All CACs with missing (aplastic) or hypoplastic (string-like) component vessels are referred to as atypical.

The issue of segmental vessel aplasia or hypoplasia raises important questions on the systems' ability to maintain adequate cerebral perfusion in normal states and the relationship between these variations and the risks of neurovascular diseases. The number of working collaterals within a CAC has been associated with lower stroke incidence in 2-year follow up studies [6, 19]. Further, variations in anatomy of the CAC have, over the years, been used to explain, in part the observed difference in prevalence, incidence and geographical distribution of certain neurovascular diseases [2].

In literature, the CAC has been classified in at least three ways namely; whole circle classification $[2,5$, $13,20]$, classification by segmental vessels $[3,4,18]$, and classification by configuration of the posterior circulation [24]. Unlike the other systems, whole circle classifications are particularly important in that they focus on both the completeness of the arterial ring and the calibre of its component vessels. In addition, whole circle studies enable comparison across populations. However, studies on whole-circle properties are sparse and when available direct comparison is often a challenge due to inconsistent study protocols, and unspecified methodologies by some researchers [4]. For instance, the definition of hypoplasia and methods of vessel measurement have varied greatly across studies.

The anatomy of the CAC has not been previously studied in Malawians and little is known on the distribution of neurovascular variations that exist in this population. Consequently, data on differences and similarities in anatomical configuration of the CAC between black Malawians and other diverse populations has not been established. This study aimed to understand and describe the anatomy of the CAC among indigenous Malawians and investigate variations in its anatomical configuration in comparison with other population groups. Knowledge of the morphological anatomy of the CAC among Malawians will help inform clinical practice, aid assessment of risks for public health interventions and offer convincing explanations on the prevalence of neuro-vascular diseases in Malawi.

\section{MATERIALS AND METHODS}

\section{Sample collection}

A total of 24 brains were studied from the recently deceased black Malawian cadavers aged 3-65 years who were candidates of autopsy for medical-legal reasons in the laboratories of the Anatomy Division, College of Medicine, University of Malawi. The brains were extracted from cranial cavities, washed with isotonic saline to remove blood around the region of interpeduncular fossa, and fixed with 10\% formalin. Cases with remarkable alteration in brain arteries and gross pathological lesions such as crush injuries, macroscopically identified tumours and severe haemorrhage were excluded from the study. 


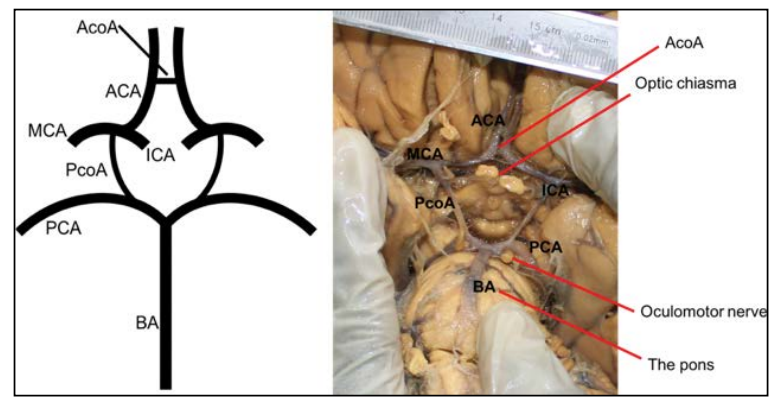

Figure 1. Schematic diagram and photograph of complete circulus arteriosus cerebri configurations showing major component vessels; AcoA - anterior communicating artery; ACA — anterior cerebral artery; MCA — middle cerebral artery; ICA — internal carotid artery; PcoA — posterior communicating artery; $\mathrm{PCA}$ — posterior cerebral artery; BA - basilar artery.

This study was conducted in accordance with the Government of Malawi Anatomy Act No. 14 of 1990 and was approved by the University of Malawi's College of Medicine Research and Ethics Committee (COMREC) with a clearance number P05/15/1729.

\section{Photography and image analysis}

Extracted brains were placed on a flat plane with a white background, ventral surface up. Images of the exposed base were taken with an 18.4 Megapixels camera (Canon EOS 600D, 2011 model, Cannon USA). Before photography, a metric calliper gauge was placed horizontally on the frontal lobes anterior to temporal poles and each image was taken perpendicularly from a $30 \mathrm{~cm}$ height in order to minimise errors that may arise due to variations in angles of view.

Photographs were initially studied under direct vision to define the completeness of the CACs, then with OSIRIS software package installed on a Microsoft Windows 10 computer to measure length and diameter of component vessels. OSIRIS is an open source extensible and portable programme for displaying, manipulating and analysing digital images. Designed in 1990 at the University Hospital of Geneva, the program is equipped with various image manipulation functions that allow a user to flip, zoom, pan and magnify images thereby allowing evaluation of distances and angles for research purposes [1]. The use of OSIRIS as a tool for studying morphometric anatomy of the CAC was described in previous works by Ansari et al. [1] and Eftekhar et al. [4], and needs not be repeated here.

Vessels studied included the AcoA, the precommunicating part of ACA (A1), the PCOA and the precom-
Table 1. Distribution of differences in whole circle properties

\begin{tabular}{lccc}
\hline Property & Variant & Subvariant & Count (\%) \\
\hline Typicality & Typical & & $6(26.09 \%)$ \\
& Atypical & Complete & $10(43.49 \%)$ \\
& & Incomplete & $7(30.43 \%)$ \\
Symmetry & Symmetrical & & $16(69.57 \%)$ \\
& Asymmetrical & & $7(30.43 \%)$ \\
\hline
\end{tabular}

municating part of PCA (P1) (Fig. 1). To be consistent with previous studies [1, 2, 12], a cut-off point of $1 \mathrm{~mm}$ was used to define vessel hypoplasia while asymmetry was defined as a vessel's diameter or length being less than half of that on the contralateral side within a CAC architecture. Data was processed in Microsoft Excel programme and CACs were schematised based on a scheme described by Riggs [20]. Descriptive statistics of component vessel properties and hypothesis tests were run in Stata version 14.

\section{RESULTS}

Data was processed at the level of the circle, where whole circle properties such as typicality, completeness and symmetry of the CAC were assessed, and at the level of individual segmental vessels, where vessel parameters such as length and diameter were analysed. One hundred and $58 \mathrm{seg}$ mental vessels from the 24 photographed arterial circles were measured and analysed in this study. There was one brain whose circle had completely lost posterior circulatory vessels (the P1 and PcoA). However, it was still included in the final analyses because its anterior circulation was intact. $69.57 \%$ of the CACs studied were complete, $37.5 \%$ of which exhibited the classical text-book (typical) configuration where all segmental vessels were intact and had diameters of at least $1 \mathrm{~mm}$. This represented an overall typicality prevalence of $26.09 \%$ (Table 1 ). Asymmetry of vessels between opposite sides of the CAC was observed in 7 (30.43\%) brains. Vessels involved in asymmetry included P1 (4), A1 (3) and PcoA (2). Two CACs had double asymmetry, one involving P1 and PcoA vessels, and the other involving $A 1$ and PcoA vessels. Table 1 presents a summary on the distribution of whole-circle CAC characteristics.

Analysis at the level of segmental vessels revealed 19 separate cases of variations. There were 7 (29.17\%) cases of vessel aplasia, 4 of which were observed on the right side of the CAC and 3 on the left. Cases of hypoplasia were seen in 5 instances on the right side 


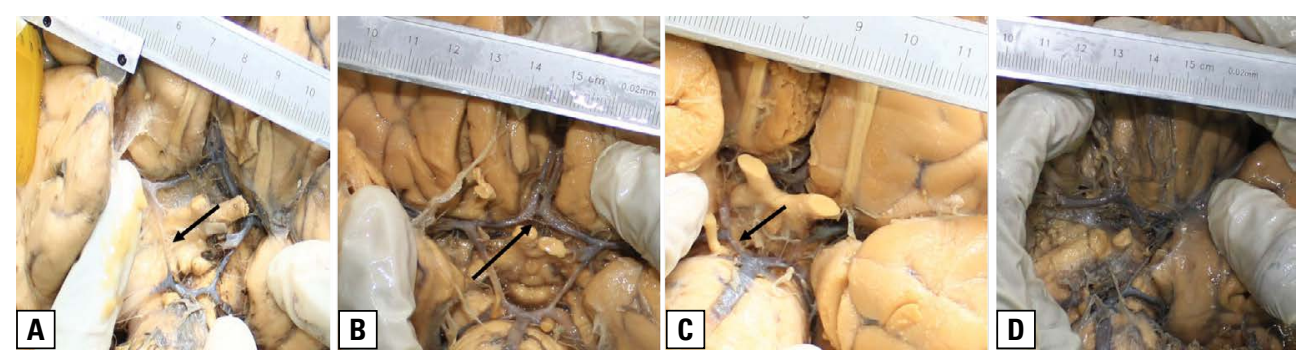

Figure 2. Some of the variations seen in this study; A. Circulus arteriosus cerebri with right unilateral posterior communicating artery hypoplasia (arrow); B. Circulus arteriosus cerebri with duplicated anterior communicating artery, the proximal of which was hypoplastic (arrow); C. Circulus arteriosus cerebri with unilateral P1 hypoplasia (arrow); D. Circulus arteriosus cerebri with right posterior communicating artery aplasia (missing).

of the CAC, 3 instances on the left and 3 instances in the AcoA, making a total of $12(45.83 \%)$ cases.

Comparison between the posterior ( $\mathrm{P} 1$ and PcoA) and anterior $(A 1, A c O A)$ circulatory routes of the CAC revealed existence of more variations in the posterior route. Eight cases of vessel hypoplasia and 7 cases of vessel aplasia were observed in the posterior circulation while only 4 cases of vessel hypoplasia were observed in the anterior. Most of the variations in the posterior circulation were associated with the PcoA. There was no case of aplasia in the anterior circulation.

Duplication of vessels, in this instance associated with the AcoA, was observed in 1 case (Fig. 2). There were only 2 candidates with more than one variation in the arterial circle (right PcoA hypoplasia with left PcoA aplasia; right PcoA and AcoA hypoplasia). The PcoA was the most variable vessel (with 12 variations) in this study, followed by the P1 (3 variations) and the AcoA (3 variations). The smallest diameter $(0.67 \mathrm{~mm})$ was observed both in AcoA and PcoA vessels. The AcoA was the shortest $(0.78 \mathrm{~mm})$ vessel while the PcoA was the widest $(7.67 \mathrm{~mm})$ and longest $(27.7 \mathrm{~mm})$ vessel. Hypoplasia was seen at least once in each vessel type (A1, P1, PcoA and AcoA) as shown in Table 2. A paired samples t-test revealed no statistically significant differences in length $(p=0.1256)$ and diameter ( $p=0.9434$ ) between first and second measurements that were collected in A1 vessel at an Alpha of 0.05. No aneurysms were observed in this study.

\section{DISCUSSION}

Whole circle properties: typicality and

\section{completeness}

Our findings suggest that although anatomically complete arterial circles are common in most populations, circles exhibiting the classical or typical configuration are not. Despite the wide prevalence range $(4.6 \%$ to $72.2 \%)$ reported in literature $[3,25]$, studies
Table 2. Variations in segmental vessels of the circulus arteriosus cerebri

\begin{tabular}{|c|c|c|c|}
\hline $\begin{array}{l}\text { Vessel } \\
\text { segment }\end{array}$ & Parameter & Laterality & $\begin{array}{l}\text { Count }(\mathrm{n}) \text { or mean } \\
\pm \text { standard deviation }\end{array}$ \\
\hline \multirow[t]{4}{*}{ AcoA } & Diameter & & $1.65 \pm 0.64$ \\
\hline & Length & & $2.92 \pm 0.94$ \\
\hline & Hypoplasia (n) & & 3 \\
\hline & Aplasia (n) & & 0 \\
\hline \multirow[t]{6}{*}{$\mathrm{A} 1$} & Diameter & & $1.75 \pm 0.45$ \\
\hline & Length & & $12.54 \pm 3.07$ \\
\hline & Hypoplasia (n) & Unilateral & 1 (left) \\
\hline & & Bilateral & 0 \\
\hline & Aplasia (n) & Unilateral & 0 \\
\hline & & Bilateral & 0 \\
\hline \multirow[t]{6}{*}{ PcoA } & Diameter & & $1.61 \pm 1.05$ \\
\hline & Length & & $12.95 \pm 4.63$ \\
\hline & Hypoplasia (n) & Unilateral & 5 (4 right, 1 left) \\
\hline & & Bilateral & 0 \\
\hline & Aplasia (n) & Unilateral & 7 (3 right, 4 left) \\
\hline & & Bilateral & 0 \\
\hline \multirow[t]{6}{*}{ P1 } & Diameter & & $1.60 \pm 1.05$ \\
\hline & Length & & $5.87 \pm 1.98$ \\
\hline & Hypoplasia (n) & Unilateral & 3 (1 right, 2 left) \\
\hline & & Bilateral & 0 \\
\hline & Aplasia (n) & Unilateral & 0 \\
\hline & & Bilateral & 0 \\
\hline
\end{tabular}

Multiple variations: 2 (right $P c o A$ hypoplasia and left $P c o A$ aplasia, right $P c o A$ and $A c o A$ hypoplasia). Duplication of vessels: $1(A \operatorname{coA}) ; A \operatorname{coA}$ — anterior communicating artery; $\mathrm{P} c 0 \mathrm{~A}$ - posterior communicating artery

that have reported typical CAC prevalences higher than $50 \%$ are rare. In the present study, the prevalence of typical CACs falls within the range and is consistent with studies conducted in other diverse populations $[3,25]$. For instance, prevalences of $14.2 \%, 27 \%, 28 \%$, and $48 \%$ were reported among Sri Lankans, Poles, Iranians and Indians, respectively [2-4, 10, 12]. Koker et al. [11], 
reported a typical CAC prevalence of $8 \%$ in Turkish population while Americans registered 19\% and 14.5\% [20]. On the contrary, complete CACs are common in several populations. In the present study, $70.83 \%$ of the brains had complete CACs. Similarly, a $90 \%$ prevalence was reported among Iranians [4]. Regardless of typicality, Zulu and Buumba [25] found completeness in $90.2 \%$ of cases among 185 Zambian cadavers, while Gunnal et al. [8], found 1 typical CAC was in a sample of 150 adult brains 90 of which had complete CACs. However, due to lack of adequate information on sample selection, demographic distribution and methods of vessel measurement, the representativeness of the findings could not be commented on.

Typicality of the arterial circle is an attribute that depends on the proposed definition for hypoplasia. Across studies, researchers have defined hypoplastic vessels varyingly. This may have contributed to the wide disparity in the prevalence of CAC typicality in literature. For instance, some authors have previously presented whole circle properties of the CAC based on estimates without actually measuring component vessel parameters such as diameter and length. Others used terms such as 'string-like', 'minute' or 'threadlike' to describe the calibre of hypoplastic vessels [3]. Moreover, even among researchers who have measured component vessels directly, the definition of hypoplasia has not been uniform. Nagawa et al. [16], used a cutoff point of $0.5 \mathrm{~mm}$ in a study of Ugandan population while lqbal used cut-off points of $0.5 \mathrm{~mm}$ in the AcoA alone and $1 \mathrm{~mm}$ in the rest of the vessels among Indians [10]. Meanwhile, Zulu and Buumba [25] did not exclude hypoplasia in the definition of typicality.

Hemodynamically-unfavourable CAC configurations were observed in this study. For instance, the case of unilateral PcoA and AcoA hypoplasia (Table 2) may affect collateral circulation of blood between the right and left arterial routes. Similarly, the case of PcoA aplasia with contralateral PcoA hypoplasia (Table 2) may affect collateral flow between the ICA and vertebral arteries systems. Both of these may be detrimental in times of cerebrovascular conditions especially where one route is affected (occluded or ruptured) and the other is required to provide backup in order to maintain adequate brain perfusion $[3,14]$. Various configurations of atypical CAC have shown to exacerbate or increase the risk of neurovascular diseases [3, 19], and their presence within a population thus demands modification in routine clinical management [7]. For instance, knowing that a larger proportion of the population exhibit CAC configurations that may inhibit communication between posterior and anterior circulatory routes or between the right and left ICAs (as may be the case in PcoA and AcoA aplasia) would mean that surgeons need to be cautious during neurovascular procedures, particularly those that involve temporary unilateral occlusion of cerebral arteries as would be the case during repair of intracranial aneurysms. Instances of bypassing or shunting effects, vascular inadequacy, recovery and lack of recovery after vascular occlusion procedures have previously been explained by variations in anatomy of cerebral arteries, particularly the CAC $[1,10]$.

\section{Vessel asymmetry}

Vessel asymmetry is a property that is scarcely reported among CAC studies. According to Ansari et al. [1], asymmetry is said to exist when the diameter of a component vessel is less than half of that of its contralateral counterpart. However, Pascalau et al. [19], used an extended definition incorporating both diameter and length. The present study used the latter.

A $30.43 \%$ prevalence of asymmetry was observed in the present study, with higher frequencies in the PcoA, followed by $A 1$ and P1 vessels (Table 1 ). The patterns of asymmetry in the CAC architecture appear to be distributed similarly across populations. Gunnal et al. [8], reported higher frequency of asymmetry in PcoA (58\%), A1 (31.33\%) and P1 (22.66\%) as compared to other vessels in Indian population, which is consistent with findings of the present study. Similar results were also observed among Turks in 2018 [19]. This seems to suggest that some variations in the anatomy of the CAC may, to some extent, be attributed to factors other than racial and geographical differences.

The issue of asymmetry causes variation in branching patterns of segmental vessels of the CAC where some segments arise from and terminate in unusual locations. This tends to position the arterial flow divider at points of bifurcation unfavourably, causing alterations in intravascular haemodynamics [10]. Using mathematical models approximating resistance to flow, vascular conductance and branching, Pascalau et al. [19] demonstrated that asymmetrical arteries offered higher resistance to blood flow and exhibited imbalanced bifurcation geometry to a higher extent than normal arteries. This has been proposed to be an independent risk factors for development of embolism and intracranial aneurysms due to higher 
possibilities for increased wall sheer stress within such vessels which may over time accelerate atherogenesis and intimal degeneration [17]. Unsurprisingly, regions with greater likelihood of aneurysms development or rupture represent the most frequently reported sites for asymmetry $[10,19]$. Moreover, A1 aplasia, PcoA hypoplasia and basilar artery asymmetrical fusion were independently associated with higher recurrence rate of saccular aneurysms following coil embolization in an 8 years cohort study [23].

\section{Aplasia and hypoplasia}

Consistent with other cadaveric studies [1, 12], the PcoA and AcoA were the narrowest vessels $(0.67 \mathrm{~mm}$ in diameter) in the present study. Segmental vessels' diameter and length are independent factors that affect functionality of the CAC as an anastomotic structure. It has been established that volume of blood flow is directly proportional to arterial diameter and inversely proportional to arterial length [16]. Thus, shorter and wider segmental vessels are seen to favour haemodynamics of the CAC to a greater extent than longer and narrower (or hypoplastic). This is analogous to the way thicker and shorter wires favour flow of current in an electric circuit. Using mathematical models, Pascalau et al. [19], observed that hypoplastic arteries presented a higher resistance to flow than otherwise normal vessels. The PcoA has been the narrowest vessel in many studies $[1,12]$. Moreover, in a normal CAC configuration, the PCOA are naturally the narrowest vessels and therefore a point of highest flow resistance in the entire CAC architecture. Due to the widely-established association between vessel hypoplasia and neurovascular diseases $[10,19,23]$, this may partly explain the higher occurrence of abnormalities in the posterior half of the CAC as observed in literature.

Apart from hypoplasia, missing vessels (aplasia) also render the CAC unfavourable as a collateral channel for arterial supply to the brain. In literature, cases of complete absence of vessels are quite rare. Most variations have been associated with string-like appearance of vessels, accessory vessels and disparities at branching [3, 19]. De Silva et al. [3] found no aplasia among 225 Sri Lankan brains. In their study, circles were visualised under a dissecting stereomicroscope which may have improved the accuracy of vessel identification, enabling the researchers to measure even the very thin vessels that could otherwise be missed under naked vision. Gunnal et al. [8], found only $2.66 \%$ aplasia cases in a sample of 150 formalin-fixed brains; however, the measurement tool was not specified. Using photography and picture manipulation computer software, the present study found aplasia in $29.17 \%$ and hypoplasia in $45.85 \%$ of the brains, reflecting fewer cases of aplasia than hypoplasia (Fig. 2). This is consistent with other cadaveric studies $[1,8,12,19]$.

In the present study, variations involving the posterior half (PcOA and P1) of the CAC were more frequent than those involving the anterior half (Table 2). This is a common finding among CAC studies across populations [8-10, 12, 19]. De Silva et al. [3] reported of these variations in $70 \%$ of 225 brains in 2009. Similarly, in a study of 100 Polish brains, Koziej et al. [12], found that $88 \%$ of the observed variations involved the PcoA alone. Using a similar method as used in the present study, Eftekhar et al. [4] found a total of $87 \%$ variations $(60 \%$ PcoA hypoplasia, $10 \%$ PcoA aplasia, and 7\% P1 hypoplasia) in the posterior half of the circle. These observations seem to suggest that the distribution of anatomical variations of the CAC may be uniform across populations.

\section{CONCLUSIONS}

The present study has provided data on whole-circle properties of the CAC among Malawians for the first time. Values of vessel diameter, vessel length as well as laterality have also been established. Variations in the anatomy of the CAC seem to exist in Malawian population and their distribution appears to be similar to those observed in other diverse populations. CAC configurations with the potential to favour atherogenesis, aneurysm formation and exacerbation of strokes have also been observed. These should be considered when performing various neurovascular procedures, particularly those that involve occlusion of main cerebral vessels in order to avoid unexpected complications. It is recommended based on this study, that such invasive neurovascular procedures be preceded by angiography and functional occlusion tests. Future studies should investigate the prevalence of neurovascular diseases, mechanisms of atherogenesis and aneurysm formation in Malawians.

\section{Acknowledgements}

The authors would like to thank the people who donated their tissues for scientific studies, and the technical staff of the Anatomy Division, Biomedical Sciences Department, College of Medicine, University of Malawi for their support. 


\section{Funding}

This work was supported by the Consortium for Advanced Research Training in Africa (CARTA). CARTA is jointly led by the African Population and Health Research Centre (APHRC) and the University of the Witwatersrand and funded by the Wellcome Trust (UK) (Grant No. 087547/Z/08/Z), the Department for International Development (DfID) under the Development Partnerships in Higher Education (DelPHE), the Carnegie Corporation of New York (Grant No. B 8606), the Ford Foundation (Grant No. 11000399), Google. Org (Grant No. 191994), Sida (Grant No. 54100029), MacArthur Foundation (Grant No. 10-95915-000-INP) and the British Council.

Part of the funding support came from the Malawi-Liverpool Wellcome Trust Clinical Research Programme through a Pre-MSc Core Training Grant awarded to $\mathrm{CN}$ under the neglected tropical diseases and non-communicable diseases research theme.

\section{Conflict of interest: None declared}

\section{REFERENCES}

1. Ansari S, Dadmehr M, Eftekhar B, et al. A simple technique for morphological measurement of cerebral arterial circle variations using public domain software (Osiris). Anat Cell Biol. 2011; 44(4): 324-330, doi: 10.5115/ acb.2011.44.4.324, indexed in Pubmed: 22254161.

2. De Silva KR, Silva $R$, Amaratunga $D$, et al. Types of the cerebral arterial circle (circle of Willis) in a Sri Lankan population. BMC Neurol. 2011; 11: 5, doi: 10.1186/14712377-11-5, indexed in Pubmed: 21241482.

3. De Silva KR, Silva R, Gunasekera WSL, et al. Prevalence of typical circle of Willis and the variation in the anterior communicating artery: A study of a Sri Lankan population. Ann Indian Acad Neurol. 2009; 12(3): 157-161, doi: 10.4103/0972-2327.56314, indexed in Pubmed: 20174495.

4. Eftekhar B, Dadmehr M, Ansari S, et al. Are the distributions of variations of circle of Willis different in different populations? Results of an anatomical study and review of literature. BMC Neurol. 2006; 6: 22, doi: 10.1186/14712377-6-22, indexed in Pubmed: 16796761.

5. El Kh, Azouzi M, Bellakhdar F, et al. Anatomical configuration of the circle of Willis in the adult studied by injection techniques. Apropos of 100 brains. Neurochirugie. 1985; 31(4): 287-293.

6. Eliasziw $M$, Henderson RD, Fox $A$, et al. Angiographically defined collateral circulation and risk of stroke in patients with severe carotid artery stenosis. Stroke. 2001; 31(1): 128-132, doi: 10.1161/01.str.31.1.128.

7. Gunnal SA, Farooqui MS, Wabale RN. Anatomical variability of the posterior communicating artery. Asian J Neurosurg. 2018; 13(2): 363-369, doi: 10.4103/ajns. AJNS_152_16, indexed in Pubmed: 29682035.

8. Gunnal SA, Farooqui MS, Wabale RN. Anatomical variations of the circulus arteriosus in cadaveric human brains. Neurol Res Int. 2014; 2014: 687281, doi: 10.1155/2014/687281, indexed in Pubmed: 24891951.
9. Hurst K, Musicki K, Molnár Z, et al. Cerebral cross-perfusion and the Circle of Willis: does physiology trump anatomy? J Vasc Diagnostics Interv. 2017; 5: 35-40, doi: 10.2147/ jvd.s121839.

10. Iqbal S. A comprehensive study of the anatomical variations of the circle of willis in adult human brains. J Clin Diagn Res. 2013; 7(11): 2423-2427, doi: 10.7860/ JCDR/2013/6580.3563, indexed in Pubmed: 24392362.

11. Koker M, Karatas A, Yilmaz H, Coban G, et al. The anatomy of circulus arteriosus cerebri (circle of willis): a study in turkish population. Turk Neurosurg. 2015; 26(1): 54-61, doi: 10.5137/1019-5149.JTN.13281-14.1, indexed in Pubmed: 26768869.

12. Koziej M, Klimek-Piotrowska W, Rybicka M, et al. A multitude of variations in the configuration of the circle of Willis: an autopsy study. Anat Sci Int. 2015; 91(4): 325-333, doi: 10.1007/ s12565-015-0301-2, indexed in Pubmed: 26439730.

13. Lazorthes G, Gouaze A, Santini J, et al. The arterial circle of the brain (circulus arteriosus cerebri). Anat Clin. 1979; 1(3): $241-257$

14. Liebeskind D. Collateral circulation. Stroke. 2003; 34(9): 2279-2284, doi: 10.1161/01.str.0000086465.41263.06.

15. McCarthy $P$, Delassus $P$, Fahy $P$, et al. An experimental investigation of the hemodynamic variations due to aplastic vessels within three-dimensional phantom models of the circle of Willis. Ann Biomed Eng. 2013; 42(1): 123-138, doi: 10.1007/ s10439-013-0905-4, indexed in Pubmed: 24018609.

16. Nagawa E, Mwaka E, Kalungi S. Bilateral hypoplasia of the posterior communicating artery: a morphological case report. Anat Physiol. 2017; 7(4): 276.

17. Nixon AM, Gunel M, Sumpio BE. The critical role of hemodynamics in the development of cerebral vascular disease. J Neurosurg. 2010; 112(6): 1240-1253, doi: 10.3171/2009.10.JNS09759, indexed in Pubmed: 19943737.

18. Ozaki T, Handa $\mathrm{H}$, Tomimoto $\mathrm{K}$, et al. Anatomical variations of the arterial system of the base of the brain. Nihon Geka Hokan (Arch Jap Chir). 1977; 46(1): 3-17, indexed in Pubmed: 561574.

19. Pascalau R, Padurean VA, Bartos D, et al. The geometry of the circle of willis anatomical variants as a potential cerebrovascular risk factor. Turk Neurosurg. 2019; 29(2): 151-158, doi: 10.5137/1019-5149.JTN.21835-17.3, indexed in Pubmed: 29484629.

20. Riggs H. Variation in form of circle of Willis. Arch Neurol. 1963; 8(1): 8, doi: 10.1001/archneur.1963.00460010024002.

21. Rosner J, Reddy V, Lui F. Neuroanatomy, Circle of Willis. [Updated 2020 Jul 31]. In: StatPearls [Internet]. Treasure Island (FL): StatPearls Publishing; 2020 Jan.

22. Siddiqi H, Ansar T, Fasih S. Variations in cerebral arterial circle of Willis in patients with hemorrhagic stroke: a computed tomography angiographic study. J Rawalpindi Med Coll. 2013; 17(2): 215-218.

23. Songsaeng $D$, Geibprasert $S$, Willinsky $R$, et al. Impact of anatomical variations of the circle of Willis on the incidence of aneurysms and their recurrence rate following endovascular treatment. Clin Radiol. 2010; 65(11): 895-901, doi: 10.1016/j.crad.2010.06.010, indexed in Pubmed: 20933644.

24. van Raamt AF, Mali WP, van Laar PJ, et al. The fetal variant of the circle of Willis and its influence on the cerebral collateral circulation. Cerebrovasc Dis. 2006; 22(4): 217-224, doi: 10.1159/000094007, indexed in Pubmed: 16788293.

25. Zulu H, Buumba PN. Anatomical Variations of the Circle of Willis as seen at the University Teaching Hospital, Lusaka, Zambia. J Prev Rehabil Med. 2017; 1(2): 61-66. 